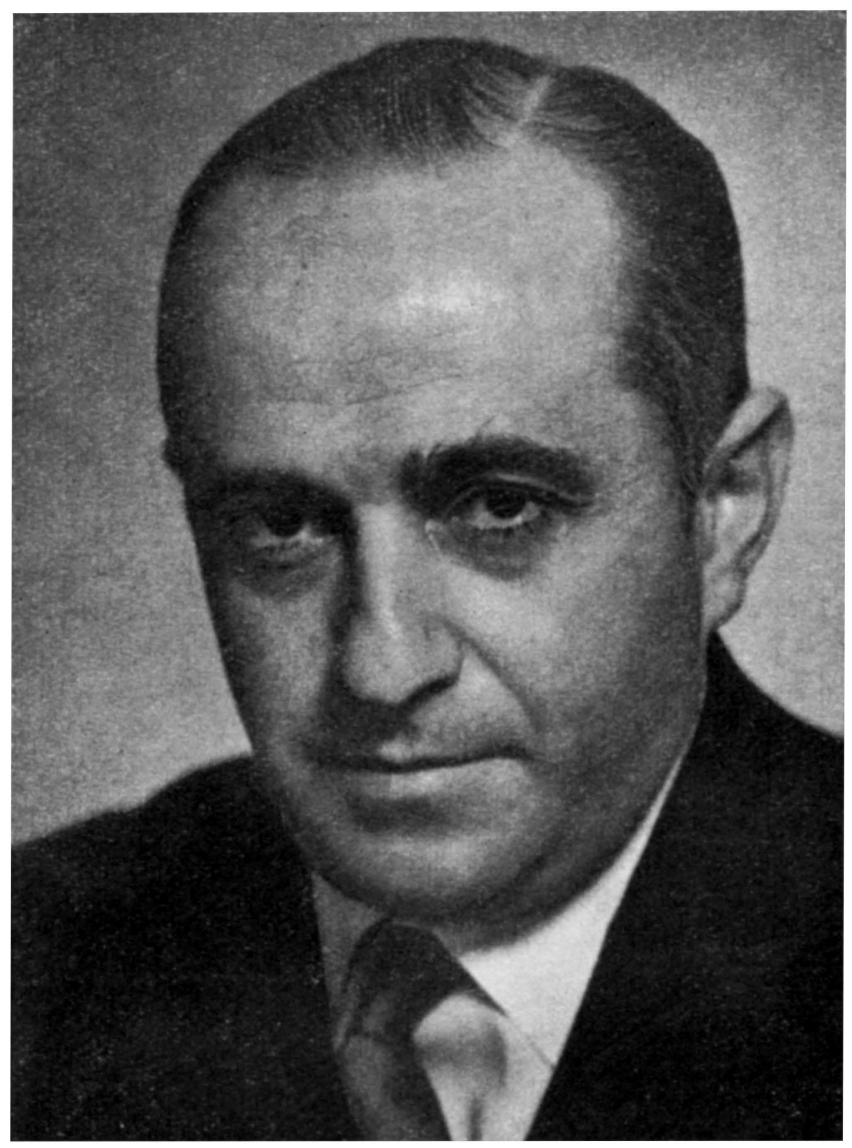

Prof, Paurino W. Loxgo 


\section{PROF. PAULINO W. LONGO}

Nascido em 7 de junho de 1903, ingressou, em fevereiro de 1920, na Faculdade de Medicina e Cirurgia de São Paulo, onde se diplomou em 1926. Em 1924 foi interno de Clínica Médica (Serviço do Prof. Ovídio Pires de Campos); em 1926 começou a trabalhar na Clínica Neurológica (Serviço do Prof. Enjolras Vampré), tendo sido nomeado assistente em 1928, ocupando êsse cargo até 1938. Foi também assistente do Prof. Enjolras Varupré na clínica privada, tendo exercido o cargo de médico interno da Secção de Moléstias Nervosas do Instituto Paulista, de que era diretor o saudoso Mesire; com o falecimento do Prof. Enjolras Vampré substituiu-o neste último cargo. Em 1938, por morte do Prof. Fausto Guerner, foi convidado para reger interinamente a Cátedra de Neurologia da Escola Paulista de Medicina; em 1939 conquistou essa Cátedra por concurso.

Por ocasião da revolta militar de 1932 foi chefe do Serviço de Neurologia de Guerra do hospital de emergência de Água Branca. Dedicou-se particularmente à malarioterapia, cuja fonte conservou por mais de 10 anos na Santa Casa de São Paulo e em outros Serviços, tendo realizado 584 inoculações. No tratamento das algias, experimentou com êxito novos recursos terapêuticos. Prestigiou o desenvolvimento da neurocirurgia, tendo dessa forma muito contribuído para o seu progresso atual em nosso meio. Como professor, participou das bancas examinadoras dos concursos para escolha dos Catedráticos de Neurologia nas Universidades da Bahia, do Rio de Janeiro, de Pernambuco e do Rio Grande do Sul; além disso, participon das bancas examinadoras dos concursos para Docência Livre em Neurologia realizadas na Faculdade de Medicina da Universidade de São Paulo, assim como daquelas designadas para julgar teses de doutoramento da mesma Cadeira.

E' membro titular da Sociedade de Medicina e Cirurgia de São Paulo desde 1931, tendo sido Presidente da Secção de Medicina Especializada em 1936; é sócio fundador da Associação Paulista de Medicina, tendo exercido os cargos de Secretário (1935) e Presidente (1937) da Secção de Neuro-Psiquiatria; foi eleito Vice-Presidente da Associação Paulista de Medicina no biênio 1947.1948; sócio correspondente da Sociedade de Medicina e Cirurgia do Rio de Janeiro; sócio honorário da Associação Médica do Instituto "Penido Burnier"; membro honorário da Secção de Neurologia da Royal Society of Medicine. de Londres (1943); membro correspondente da Sociedade Brasileira de Neurologia, Psiquiatria e Medirina Legal do Rio de Janeiro (1938); sócio honorário da Asociación Médica Argentina, Buenos Aires (1946); membro honorário da Sociedad Chilena de Neurologia, Psiquiatria y Neurocirugia (1942); membro corresponden- 
te da Facultad de Biologia y Ciencias Médicas, Universidad de Chile (1947); membro da Sociedad Médica de Santiago, Chile (1947); membro correspondente da Sociedad de Neurologia y Neurocirugia de Montevidéo (1951); membro da Societé Française de Neurologie, Paris.

Participou da Semana Oftalmológica em São Paulo (1927); do I Congresso Médico Paulista (1933); da II Conferência Latino-Americana de Neurologia, Psiquiatria e Medicina Legal (1930); do II Congresso Médico Paulista (1936); da Reunião Neurológica de Campinas (1936); da Semana Neurológica de Belo Horizonte (1937); do II Congresso Interamericano de Neurocirurgia (Santiago do Chile, 19.17); do I Congresso Neurológico Internacional (Berna, 1931) e do II Congresso Neurológico Internacional (Londres, 1935). Delegado oficial ao Congresso de Psicocirurgia (Lisboa, 1948) e ao V Congresso Internacional de Neurologia (Lisboa, 1953).

TRABALHOS PUBLICADOS

1. Esclerose lateral amiotrófica. Forma pseudo-polinevrítica. Bol. Soc. Med. e Cir. São Paulo, $9: 279-280,1926$.

2. Contribuição ao Estudo da Esclerose Lateral Amiotrófica. Tese inaugural, Faculdade de Medicina da Universidade de São Paulo, 1926.

3. Compressão da medula por tumor (em col. com Li. Vainpré). Bol. Soc. Med. e Cir. São Paulo, 9:104-106, 1926.

4. Malarioterapia da paralisia geral (em col. com Linjolras Vampré). Bol. Soc. Med. e Cir. São Páulo, 10:155-160, 1927.

5. Tumor justamedular intradural (em col. com E. Vampré e A. C. Camargo). Bol. Soc. Med. e Cir. São Paulo, 12:3-6, 1928.

6. Artropatia polinevrítica (em col. com E. Vimpré). Bol. Soc. Med. a Cir. São Paulo, 13:2066-2̌̈o, 1929.

7. Formas clínicas do cancro vertebral (em col. com E. Vampré). Bol. Soc. Med. e Cir. São Paulo, 13:292-310, 1929.

8. Efeitos tardios da intoxicação oxicarbonada. Bol. Soc. Med. e Cir. São laulo, 14:509-510, 1930 .

9. Esclerose Lateral Amiotrófica. Forma l'studo-polinevrítica dos Membros Superiores (Poliomielite Superior). 'Tese apresentada à Sociedade de Medicina e Cirurgia de São Paulo, 1931.

10. Formas atípicas da esclerose lateral amiotrótical. Bol. Soc. Med. e Cir. Sũo Paulo, 15:25-35, 1931 .

11. Nouvelte contribution: à l'étude des formes exceptionelles de sclérose latérele anyotrophique (em col. zom E. Vampré). Rev. Sud-Amer. de Méd. et dc Chir , 2:608-625, 1931.

12. Conseqüiências oculares da vacinação anti-rúbica (em col. com ki. Vampré e W. Belfort de Mattos). Bol. Soc. Med. e Cir. São Paulo, 10:274-285, 1927.

13. Sutura dos nervos mediano e cubital. Cura (em col. con Eurico Bastos). Anais do I Congresso da Associação Paulista de Medicina, 1933.

14. Paralisia do nervo crural traumática. Évolução e cura (em col. com Eurico Bastos). Anais do 1 Congresso da Associação Paulista de Medicina, 1933.

15. Camptocormia: patogenia e ternpêutica. Bol. Soc. Med. e Cir. São Paulo, $17: 27-32,1933$.

16. Formes atypiques de la maladie de Charcot (em col. com E. Vampré). Rev. Sud-Amer. de Méd. et de Chir., 4:251-262, 1933. 
17. Considerações em tôrno de três casos graves de espasmofilia. Pediat. Prát. (São Paulo), 4:447-456, 1933.

18. Fraturas da abóbada craniana na infância (em col. com O. Pinto de Souza) Pediat. Prát. (São Paulo), 5:49-53, 1934.

19. Lei de Bastian: critica e valor em face dos traunatismos graves da medula. Rev. Neurol. e Psychiat. São P’aulo, 1:24-30, 1934.

20. Psicoses puerperais (em col. com E. Vampré). Bol. Soc. Med. e Cir. São Paulo, 15:506, 1932.

21. 'Tumores múltipłos do eixo cérebro-espinhal (em. col. com E. Vampré, A. 'Tolosa, H. S. Minalin, O. Lange e J. Ribeiro do Vale). São Paulo Méd., 6:175-200, 1933 .

22. Alterações graves da sensibilidade na esclerose lateral amiotrófica. Rev. Neurol. e Psychiat. São Paulo, 2:32-39, 1936.

23. Resultados clínicos em 10 anos de malarioterapia. Bol. Soc. Med. e Cir. São Paulo, 21:2-3, 1937.

24. Resultados da malarioterapia na atrofia óptica luética. Arq. Inst. Penido Burnier (Campinas), 4:183-206, 1936.

25. Paralisias periódicas. Rev. Neuroi. e Psychiat. São Paulo, 2:336-346, 1936.

26. Alcoolização intra-raquidiana em 10 casos de nevralgias rebeldes de origem neoplísica. Rev. Assoc. Paulista de Med., 10:28-29, 1937.

27. Tratamento psicogaliânico dos psiconeuróticos de guerra, sinistrosidos e pitiáticos São Paulo Méd., 10:259-270, 1937.

28. 'Traumatismos cranianos na infância (em col. com O. Pinto de Souza). Anais do II Congresso da Associação Paulista de Medicina, 1938.

29. Psamoma da região rolândica propagando-se à região parietal dircita. Valor semiológico das atrofias musculares e assimbolia táctil nas lesões do lobo parietal (em col. com Carlos Gama). São Paulo Méd., 11:107-133, 1938.

30. Dois casos de amaurose pitiática curados pelo método psicogalvânico (em col com F. Oliveira Bastos). Rev. Assoc. Paulista de Med., 12:187-194, 1938.

31. Éritromelagia entre as perturbações vaso-motoras. Tratamento pelos raios $\mathrm{X}$ (em col. com M. Roxo Nobre). Rev. Assoc. Paulista de Med., 13:121, 1938.

32. A propósito de um caso de miastenia de Erb-Goldflam. Rev. Neurol. e Psychiat. São Paulo, 4:106-113, 1938.

33. Alterações da Sensibilidade na Paralisia Periódica Fanniliar de Westphal. 'lese apresentada no concurso para Professor Catedrático de Clínica Neurológica na Escola Paulista de Medicina, 1939.

34. Síndrome de Avelis e síndrome de Claude Bernard-Horner de etiologia obscura (em col. com F. Oliveira Bastos e J. Paula Assis). Therapia, 1:3-7, 1939.

35. Nevralgia essencial do glossofaríngeo. Neurotomia intracraniana (em col. com A. Mattos Pimenta e P. Pinto Pupo). Rev. Assoc. Patulista de Med., 16: $320,1940$.

36. Síndrome parietal puro por embolia arterial (em col. com P. P'into Pupo e A. Marques). Rev. Paulista de Med., 17:15:, 1940.

37. Sôbre um caso de síndrome compressivo da cauda equina de provável causa luética (em col. com O. Lemmi). Rev. Paulista de Med., 17:209, 1940.

38. O Dilantin na epilepsia. Sôbre alguns casos de intoxicação (em col. com $O$. Lemmi). Rev. Paulista de Med., 19:299, 1941.

39. Aracnoidite espinal adesiva (em col. com J. Ribeiro do Vale). Rev. Neurol. e Psychiat. São Paulo, 8:192-193, 1942.

40. Intestinos em Neurologia. Rev. Neurol. e Psychiat. São Paulo, 8:243-251, 1942.

41. Neurose gástrica e insulinoterapia (ein col. com J. Arruda). Rev. Paulista de Med., 21:286-289, 1942. 
42. Prognóstico e terapêtutica na epilepsia. Rev. Paulista de Med., 21:84-102, 1942.

43. Tratamento das Psiconeuroses de Guerra. Edigraf, Săo P’aulo, 1913.

44. Possivel parentesco entre as moléstias familiares com lesões predominantes na medula e moléstias familiares com lesões no neurônio periférico (em col. com 1. Pinto Pupo e D. Giorgi). Arq. Neuro-Psiquiat., 1:27-43, 1943.

45. O exame do líquiido cefalorraquidiano (em col. com J. Baptista dos Reis) Med., Cir. e Farm., $\mathrm{n}^{\circ}$ 91, págs. 497-529, 1943.

46. Pneumatocele por traumatismo da região frontal (em col. com A. Mattos Pimenta e F. Prudente de Aquino). Arq. Neuro-Psiquiat., 3:52-60, 145.

4.7. Síndrome do lobo parietal. Apraxia isolada do membro superior esquerdo (em col. com P. Pinto Pupo). São Paulo Méd., 15:298-311, 1945.

48. Considerações a propósito de 5 casos de afasia motora (em col. com A. B. Lefèvre). A rq. Neuro-Psiquit., 3:249-257, 1945.

49. Penicilinoterapia em neuroluéticos já malarizados (em col. com M. Robortella e J. Baptista dos Reis). Arq. Neuro-Psiquiat., 4:47-54, 1946.

50. Penicilinoterapia em um caso de neurite óptica luética (em col. com J. M. Bittencourt). A rq. Neuro-Psiquiat., 4:55-58, 1946.

51. 'Tumor da região do $3^{\circ}$ ventrículo. Diagnóstico eletrencefalográfico (em col. com P. Pinto Pupo, A. Mattos Pimenta e O. Lemmi). Arq. Neuro-Psiquiat., $6: 169-177,1946$.

52. Psicoses tóxicas consequientes à quinacrina (em col. com J. Arruda). Arq Neuro-Psiquiat., 6:159-168, 1946.

53. Considerações sôbre um caso de meningite por bacilo de l’feifer. Rev. Paulista de Med., 30:48, 1947.

54. Enxaqueca oftalmoplégica. Estudo clínico e eletrencefalográfico de 2 casos (em col. com P. P'into P'upo e O. I emmi). Arq. Neuro-Psiquiat., 6:178-188, 1947.

55. A convulsoterapia nos estados catatônicos (em col. com F. Oliveira Bastus). Rev. Paulista de Med., 16:387, 1948.

56. Lobotomia pré-frontal. Resultados clínicos em hospital privado (em col. com A. Mattos Pimenta e J. Arruda). Arq. Neuro-Psiquiat., 7:1411-155, 1949.

57. Pressão do líquiido cefalorraqueano. Conceito de normalidade (em col. coin J. V. Dourado e J. Baptista dos Reis). Rev. Paulista de Med., 36:4.5-48, 1950.

58. Résultats électroencéphalographiques dans 33 cas de tumeurs intracraniennes chirurgicalement verifiées (em col. com P. Pinto Pupo, O. Pazzanese $\in$ A. Mattos Pimenta). Arq. Neuro-P'siquiat., 8:43-46, 1950.

59. Diagnóstico e orientação terapêutica na epilepsia (em col. com P. Pinto Pupo e O. Pazzanese). Rev. Paulista de Med., 39:417-439, 1951.

60. A eletrocorticografia como meio de orientação na terapêutica cirúrgica da epilepsia (em col. com P. Pinto Pupo, O. Pazzanese, $\Lambda$. Mattos Pimenta e O. Lemmi). A rq. Neuro-Psiquiat., 10:365-373, 1952.

61. Expérience personelle sur la thérapentique et le diagnostic de lancurysme artério-veineux intracrânien (em col. com A. Mattos Pimenta e C. Pareira da Silva). Anais do $\mathrm{V}$ Congresso Neurológico Internacional (Lisboa), 2:65,66, 1953.

62. Considerações sôbre mil pacientes com distúrbios paroxísticos suspeitos de epilepsia (em col. com P. Pinto Pupo, O. Pazzanese e B. A. Sampaio). Rev. Paulista de Med., $42: 376,1953$. 